\title{
Identification of Neisseria gonorrhoeae by the Bruker Biotyper Matrix- Assisted Laser Desorption Ionization-Time of Flight Mass Spectrometry System Is Improved by a Database Extension
}

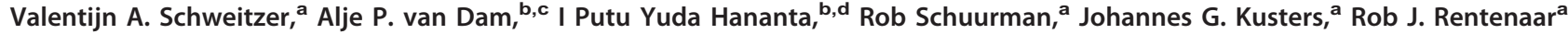 \\ Department of Medical Microbiology, University Medical Centre Utrecht, Utrecht, The Netherlands a'; Public Health Laboratory, Cluster of Infectious Diseases, Health \\ Service of Amsterdam, Amsterdam, The Netherlands' ; Department of Medical Microbiology, Onze Lieve Vrouwe Gasthuis General Hospital, Amsterdam, The \\ Netherlandsc; Department of Dermatology, Faculty of Medicine, Universitas Gadjah Mada, Yogyakarta, Indonesiad
}

\begin{abstract}
Identification of Neisseria gonorrhoeae by the Bruker matrix-assisted laser desorption ionization-time of flight mass spectrometry (MALDI-TOF MS) system may be affected by "B consistency categorization." A supplementary database of $17 \mathrm{~N}$. gonorrhoeae main spectra was constructed. Twelve of $64 \mathrm{~N}$. gonorrhoeae identifications were categorized with B consistency, which disappeared using the supplementary database. Database extension did not result in misidentification of Neisseria meningitidis.
\end{abstract}

$\mathrm{M}$ atrix-assisted laser desorption ionization-time of flight mass spectrometry (MALDI-TOF MS) yields highly reliable, rapid bacterial identifications. The Bruker MALDI-TOF MS system uses the best database match of a mass spectrum for identification. MALDI-TOF MS may misidentify species within closely related bacteria such as the Streptococcus mitis, Enterobacter cloacae, Acinetobacter baumannii-Acinetobacter calcoaceticus, Bacillus cereus, and Burkholderia cepacia complexes due to highly similar mass spectra (1-5). The Bruker Biotyper MALDI-TOF MS system adds a "category B consistency" warning when the list of top 10 database matches contains different species of the same genus with score values of $\geq 2.000$. We and others observed that B consistency categorizations of Neisseria gonorrhoeae isolates frequently occur with Neisseria meningitidis score values of $\geq 2.000$ (6). B consistency categorizations indicate uncertainty about the true identification of the organisms, which is of concern because $N$. gonorrhoeae and N. meningitidis may be isolated from the same body sites (7). Misidentification of $N$. gonorrhoeae can have implications for diagnosis in patients and their sexual partners and for public health. An extension of the database may improve the MALDI-TOF MS identification results (8). We tested whether a database extension of $17 \mathrm{~N}$. gonorrhoeae main spectra (MSPs) (NG17) superimposed on the MBT-BDAL-5627 MSP library (5627) resolved B consistency categorizations in N. gonorrhoeae identifications without affecting N. meningitidis identifications using the Bruker Biotyper system. Eighty-one N. gonorrhoeae unrelated (i.e., one isolate per individual patient) isolates were collected (see Table S1 in the supplemental material). N. gonorrhoeae isolates were confirmed as N. gonorrhoeae by the Cobas 4800 CT/NG PCR (Roche Molecular Diagnostics, Almere, The Netherlands), performed according to the manufacturer's instructions. Quality control (QC) strains from the Gonococcal Resistance against Antibiotics Surveillance project (GRAS) QC distribution, which is a national surveillance program for N. gonorrhoeae resistance, were not reconfirmed by PCR. Seventeen isolates were used for construction of the supplementary NG17 MSP library (see Table S1). Sixty-four N. gonorrhoeae isolates were used to test the databases. Thirty N. meningitidis isolates were collected at the University Medical Centre Utrecht (UMCU) and isolated from either blood $(n=18)$ or cerebrospinal fluid $(n=11)$ or from a quality control distribution $(n=1) . N$. meningitidis isolates from invasive meningococcal disease were confirmed by The Netherlands Reference Laboratory for Bacterial Meningitis, Amsterdam, The Netherlands (Gram-negative diplococci, gamma-glutamyl aminopeptidase positive, reacting with one of the serogrouping antibodies, typable using fetA and porA molecular typing methods). Twenty-seven of $30 \mathrm{~N}$. meningitidis isolates were also tested and found negative in the N. gonorrhoeae PCR. Direct transfer preparation was performed in duplicate on polished steel target plates (Bruker, Bremen, Germany) after a 1- or 2-day incubation (depending on whether sufficient biomass was harvested from a colony) on chocolate agar (chocolate agar GC II Agar with IsoVitaleX, 254060; BD, NJ, USA) under 5\% $\mathrm{CO}_{2}$, using a sterilized wooden toothpick. $\alpha$-Cyano-4-hydroxycinnamic acid (HCCA) matrix (Bruker, Bremen, Germany) was applied, and the plates were air dried. Analyses were performed using the Bruker Microflex MALDI mass spectrometer, Biotyper software, and the MBT-BDAL-5627 MSP library (5627) (Bruker) according to the manufacturer's instructions (9). Acquired spectra were reanalyzed with the NG17 database extension to the 5627 database (5627+NG17).

MSPs were generated after a 2-day incubation as described previously (8). MSPs were created using 240 shots with a maximum of 80 shots per raster spot. Eight target spots were used per isolate, and 3 spectra were acquired per target spot. Subsequently,

Received 12 January 2016 Returned for modification 13 January 2016 Accepted 13 January 2016

Accepted manuscript posted online 13 January 2016

Citation Schweitzer VA, van Dam AP, Hananta IPY, Schuurman R, Kusters JG, Rentenaar RJ. 2016. Identification of Neisseria gonorrhoeae by the Bruker Biotyper matrix-assisted laser desorption ionization-time of flight mass spectrometry system is improved by a database extension. J Clin Microbiol 54:1130-1132. doi:10.1128/JCM.00016-16

Editor: E. Munson

Address correspondence to Rob J. Rentenaar, rrentenaar@yahoo.com. Supplemental material for this article may be found at http://dx.doi.org/10.1128 /JCM.00016-16

Copyright @ 2016, American Society for Microbiology. All Rights Reserved. 
TABLE 1 N. gonorrhoeae MALDI-TOF score values are increased and B consistency categorizations are eliminated by database extension with 17 N. gonorrhoeae main spectra

\begin{tabular}{|c|c|c|c|}
\hline Measurement & Database 5627 & Database 5627 + NG17 & $P$ \\
\hline No. of N. gonorrhoeae isolates & 64 & 64 & \\
\hline $\begin{array}{l}\text { Score value of the top database match (with N. gonorrhoeae } \\
\text { MSP) (mean [range]) }\end{array}$ & $2.259(1.875-2.453)$ & $2.436(1.974-2.700)$ & $<0.05^{a}$ \\
\hline $\begin{array}{l}\text { B consistency due to any database match among the top } 10 \text { with } \\
N \text {. meningitidis MSP with score value of } \geq 2.000 \text { (no. }[\%] \text { ) }\end{array}$ & $12 / 63(19)$ & $0 / 63(0)$ & $<0.05^{b}$ \\
\hline $\begin{array}{l}\text { Score value of the best database match with } N \text {. meningitidis } \\
\text { (mean [range]) }\end{array}$ & $1.931(1.544-2.133)$ & $\mathrm{NA}^{d}$ & \\
\hline $\begin{array}{l}\text { B consistency due to database match among the top } 2 \text { with } \\
N \text {. meningitidis MSP with score value of } \geq 2.000 \text { (no. [\%]) }\end{array}$ & $7 / 63(11)$ & $0 / 63(0)$ & $<0.05^{b}$ \\
\hline
\end{tabular}

\footnotetext{
${ }^{a}$ Wilcoxon signed-rank test.

${ }^{b}$ McNemar test for paired proportions.

${ }^{c}$ One isolate was identified to the genus level only (N. gonorrhoeae score value of 1.875).

${ }^{d} \mathrm{NA}$, not applicable.
}

a maximum of the 3 most aberrant spectra from these $24 \mathrm{spec}-$ tra were deselected for MSP creation. MSP dendrograms were constructed using Biotyper 3.1 software. Results obtained with the 5627 database versus those with the $5627+$ NG17 database were analyzed using the Wilcoxon signed-rank test for score value results (Graph Pad Prism software version 6.02) or the McNemar test for B consistency categorization results (SPSS version 21).

Using the best database match (with a score value of $\geq 2.000$ ), MALDI-TOF MS identified 63 of $64 \mathrm{~N}$. gonorrhoeae isolates correctly to the species level (Table 1; see also Fig. S1A in the supplemental material). The best matches were almost uniquely $(62 / 64 ; 97 \%$ of tested isolates) with a single entry from the 5627 database, i.e., N. gonorrhoeae MSP ATCC 49226 THL. In the 2 remaining isolates, the best match was with MSP N. gonorrhoeae ch012 PGM. One isolate was identified to the genus level only (best match with the 5627 database: N. gonorrhoeae score value of 1.875) (Table 1). All 64 identifications were confirmed by N. gonorrhoeae-specific PCRs (see Table S1 in the supplemental material). B consistency categorization occurred in $19 \%$ of $N$. gonorrhoeae isolates using the 5627 database (Table 1). Notably, in $11 \%$ of $N$. gonorrhoeae isolates with score values of $\geq 2.000$, a $N$. meningitidis MSP with a score value of $\geq 2.000$ occurred as the second-best database match (Table 1). In a MSP dendrogram, all 17 newly created $N$. gonorrhoeae main spectra clustered together with a single 5627 MSP, N. gonorrhoeae ATCC 49226 THL (see Fig. S2 in the supplemental material). The 6 other N. gonorrhoeae MSPs from the 5627 database were more distantly related to the ATCC 49226 THL entry and the NG17 MSPs. Analyses using the $5627+$ NG17 database resulted in significantly increased score values and complete disappearance of B consistency categorizations in N. gonorrhoeae isolates (Table 1; see also Fig. S1 in the supplemental data). The best database matches were with the newly created $17 \mathrm{~N}$. gonorrhoeae MSPs and not with the MSPs from the 5627 database (data not shown). In N. meningitidis isolates, no B consistency categorizations were found using either the 5627 or the $5627+$ NG17 database. The score values of the best database matches were unaffected by addition of the NG17 MSPs (see Fig. S1B and Table S2 in the supplemental material).
Nucleic acid amplification tests (NAAT) have become the cornerstone in the diagnosis of genital as well as many extragenital $N$. gonorrhoeae infections. However, at present, NAATs alone are insufficient for accurate detection of antibiotic resistance in cases of treatment failure. Therefore, culturing of $N$. gonorrhoeae remains an important adjunct to NAATs (10-13). We found that MALDITOF MS N. gonorrhoeae identification was highly reliable, with abrogation of B consistency categorizations by extension of the database. Reliable MALDI-TOF MS identification of N. gonorrhoeae, using the best database match, was also demonstrated by others $(6,14-16)$. Nevertheless, Bruker has not yet received FDA approval for N. gonorrhoeae identification using the MALDI Biotyper CA system (see http://www.accessdata.fda.gov/cdrh_docs /pdf14/k142677.pdf).

Previously, B consistency categorizations were reported in 4/5 $N$. gonorrhoeae isolates tested (6). In our study, the disappearance of $\mathrm{B}$ consistency categorization resulted from $\geq 10$ database matches with $N$. gonorrhoeae MSPs with higher score values than the matches with $N$. meningitidis MSPs from the 5627 library, effectively displacing the $N$. meningitidis MSPs from the list of the top 10 database matches. With the 5627 database only, the best matches were with Neisseria gonorrhoeae ATCC 49226 THL MSP in 62 of 64 identifications. Also, this single MSP was found to closely cluster with the newly constructed NG17 MSPs in an MSP dendrogram. Moreover, this MSP was distantly related to the 6 other N. gonorrhoeae MSPs from the 5627 database. By merging the 5627 and NG17 databases, 2 clusters of MSPs appear (see Fig. S2 in the supplemental material): one cluster of MSPs, likely from the Ilina study, representing different Por types from Russian clinical isolates and another cluster with the ATCC 49226 THL MSP that clustered together with the NG17 MSPs (16). Subtle differences in factors such as the media used, the incubation conditions, and the instrument settings might influence $N$. gonorrhoeae isolates to better match our locally produced MSPs.

In conclusion, $N$. gonorrhoeae score values are improved and B consistency categorizations of $N$. gonorrhoeae identifications can be abrogated by the NG17 database extension without affecting $N$. meningitidis identification. 


\section{ACKNOWLEDGMENTS}

We thank Nuria Roca, Judith Vlooswijk, and Peter van Bodegom for technical help and L. Spanjaard from The Netherlands Reference Laboratory for Bacterial Meningitis for advice on meningococcal identification.

\section{REFERENCES}

1. Fehlberg LC, Andrade LH, Assis DM, Pereira RH, Gales AC, Marques EA. 2013. Performance of MALDI-ToF MS for species identification of Burkholderia cepacia complex clinical isolates. Diagn Microbiol Infect Dis 77:126-128. http://dx.doi.org/10.1016/j.diagmicrobio.2013.06.011.

2. Hsueh PR, Kuo LC, Chang TC, Lee TF, Teng SH, Chuang YC, Teng LJ, Sheng WH. 2014. Evaluation of the Bruker Biotyper matrix-assisted laser desorption ionization - time of flight mass spectrometry system for identification of blood isolates of Acinetobacter species. J Clin Microbiol 52: 3095-3100. http://dx.doi.org/10.1128/JCM.01233-14.

3. van Veen SQ, Claas EC, Kuijper EJ. 2010. High-throughput identification of bacteria and yeast by matrix-assisted laser desorption ionizationtime of flight mass spectrometry in conventional medical microbiology laboratories. J Clin Microbiol 48:900-907. http://dx.doi.org/10.1128 /JCM.02071-09.

4. Alatoom AA, Cunningham SA, Ihde SM, Mandrekar J, Patel R. 2011. Comparison of direct colony method versus extraction method for identification of Gram-positive cocci by use of Bruker Biotyper matrix-assisted laser desorption ionization - time of flight mass spectrometry. J Clin Microbiol 49:2868-2873. http://dx.doi.org/10.1128/JCM.00506-11.

5. Patel R. 2013. Matrix-assisted laser desorption ionization-time of flight mass spectrometry in clinical microbiology. Clin Infect Dis 57:564-572. http://dx.doi.org/10.1093/cid/cit247.

6. Khot PD, Couturier MR, Wilson A, Croft A, Fisher MA. 2012. Optimization of matrix-assisted laser desorption ionization-time of flight mass spectrometry analysis for bacterial identification. J Clin Microbiol 50:3845-3852. http://dx.doi.org/10.1128/JCM.00626-12.

7. Young H, Bain SS. 1983. Neisserial colonisation of the pharynx. Br J Vener Dis 59:228-231.

8. Schulthess B, Bloemberg GV, Zbinden R, Bottger EC, Hombach M. 2014. Evaluation of the Bruker MALDI Biotyper for identification of Gram-positive rods: development of a diagnostic algorithm for the clinical laboratory. J Clin Microbiol 52:1089-1097. http://dx.doi.org/10.1128 /JCM.02399-13.
9. de Jong E, de Jong AS, Smidts-van den Berg N, Rentenaar RJ. 2013. Differentiation of Raoultella ornithinolytica/planticola and Klebsiella oxytoca clinical isolates by matrix-assisted laser desorption/ionization - time of flight mass spectrometry. Diagn Microbiol Infect Dis 75:431-433. http: //dx.doi.org/10.1016/j.diagmicrobio.2012.12.009.

10. Low N, Unemo M, Skov Jensen J, Breuer J, Stephenson JM. 2014. Molecular diagnostics for gonorrhoea: implications for antimicrobial resistance and the threat of untreatable gonorrhoea. PLoS Med 11:e1001598. http://dx.doi.org/10.1371/journal.pmed.1001598.

11. Centers for Disease Control and Prevention. 2012. Update to CDC's sexually transmitted diseases treatment guidelines, 2010: oral cephalosporins no longer a recommended treatment for gonococcal infections. MMWR Morb Mortal Wkly Rep 61:590-594.

12. Bignell C, Unemo M. 2013. 2012 European guideline on the diagnosis and treatment of gonorrhoea in adults. Int J STD AIDS 24:85-92. http: //dx.doi.org/10.1177/0956462412472837.

13. Centers for Disease Control and Prevention. 2014. Recommendations for the laboratory-based detection of Chlamydia trachomatis and Neisseria gonorrhoeae-2014. MMWR Recomm Rep 63:1-19.

14. Golparian D, Dauphin B, Hellmark B, Claesson B, Unemo M. 2013. P2.046 Evaluation of matrix-assisted laser desorption/ionization time-offlight mass spectrometry (MALDI-TOF-MS) for species identification within the Neisseria genus-effective alternative to conventional techniques? Sex Transm Infect 89(Suppl 1):A102. http://dx.doi.org/10.1136 /sextrans-2013-051184.0311.

15. Branda JA, Rychert J, Burnham CA, Bythrow M, Garner OB, Ginocchio CC, Jennemann R, Lewinski MA, Manji R, Mochon AB, Procop GW, Richter SS, Sercia LF, Westblade LF, Ferraro MJ. 2014. Multicenter validation of the Vitek MS v2.0 MALDI-TOF mass spectrometry system for the identification of fastidious gram-negative bacteria. Diagn Microbiol Infect Dis 78:129-131. http://dx.doi.org/10.1016/j.diagmicrobio .2013.08.013.

16. Ilina EN, Borovskaya AD, Malakhova MM, Vereshchagin VA, Kubanova AA, Kruglov AN, Svistunova TS, Gazarian AO, Maier T, Kostrzewa M, Govorun VM. 2009. Direct bacterial profiling by matrixassisted laser desorption-ionization time-of-flight mass spectrometry for identification of pathogenic Neisseria. J Mol Diagn 11:75-86. http://dx .doi.org/10.2353/jmoldx.2009.080079. 